\title{
PROJETO MECÂNICO DE UM TÚNEL DE VENTO PARA TESTES AERODINÂMICOS
}

\author{
EM LABORATÓRIO
}

MECHANICAL DESIGN OF A WIND TUNNEL FOR AERODYNAMIC TESTS IN THE

\author{
LABORATORY
}

Juliano Leite de Almeida ${ }^{1}$

Eduardo Bueno ${ }^{2}$

Márcio Kawamura ${ }^{3}$

\section{RESUMO}

A utilização de aerogerador está em plena expansão e as pesquisas no campo aerodinâmico são fundamentais, uma vez que a aplicação de túneis de vento é uma pratica bastante eficaz no projeto de pás. O túnel de vento é um equipamento utilizado em testes aerodinâmicos para verificar a eficiência, resistência ou capacidade de uma parte ou mesmo de um todo sem a necessidade de haver movimento dos objetos, apenas o vento percorre a seção de testes e atinge o modelo. Sabe-se que a aplicação de túneis de vento em laboratórios de projeto para ensaio de sistemas aerodinâmicos é um recurso muito eficaz nas pesquisas e testes dos protótipos de pás e aerogeradores, pois ensaiar protótipos nas condições de vento ao ar livre não é muito eficaz, pelo fato de depender das condições climáticas e da velocidade do vento local que não é constante, dificultando o monitoramento e coleta de informações para determinar a eficiência de um protótipo. Esta pesquisa apresenta o desenvolvimento do projeto mecânico de um túnel de vento, visando desenvolver tecnologia na área de aerogeradores, bem como para realizar testes aerodinâmicos.

Palavras-Chave: Aerogerador. Pás. Protótipo. Dimensionamento.

\footnotetext{
1 Acadêmico de Engenharia Mecânica da Universidade Alto Vale do Rio do Peixe (UNIARP).

2 Mestre em Universidade Federal de Santa Catarina (UFSC). Professor da Engenharia Elétrica e Engenharia Mecâncica da Universidade Alto Vale do Rio do Peixe (UNIARP). Email: bueno@uniarp.edu.br.

3 Coordenador e Professor da Engenharia Mecânica da Universidade Alto Vale do Rio do Peixe (UNIARP). Email: marciokawamura@gmail.com.
} 


\section{ABSTRACT}

The use of wind turbine is in full expansion and research in the aerodynamic field is fundamental, since the application of wind tunnels is a very effective practice in the design of blades. The wind tunnel is an equipment used in aerodynamic tests to check the efficiency, strength or capacity of a part or even a whole without the need for movement of the objects, only the wind travels through the test section and reaches the model. It is known that the application of wind tunnels in aerodynamic systems testing laboratories is a very effective resource in the research and testing of blade and wind turbine prototypes, because testing prototypes in open-air wind conditions is not very effective, because it depends on weather conditions and local wind velocity that is not constant, making it difficult to monitor and collect information to determine the efficiency of a prototype. This research presents the development of the mechanical design of a wind tunnel, aiming to develop technology in the field of aerogenerators, as well as to perform aerodynamic tests.

Keywords: Wind turbine. Blades. Prototype. Sizing.

\section{INTRODUÇÃO}

Túnel de vento é um equipamento utilizado em testes aerodinâmicos para verificar a eficiência, resistência ou capacidade de uma parte ou mesmo de um todo sem a necessidade de haver movimento dos objetos, apenas o vento percorre a seção de testes [1].

Sabe-se que a aplicação de túneis de vento em laboratórios de projeto para ensaio de sistemas aerodinâmicos é um recurso muito eficaz nas pesquisas e testes dos protótipos de pás e aerogeradores, pois segundo Okamoto et al. [1] ensaiar protótipos nas condições de vento ao ar livre não é muito eficaz, pelo fato de depender das condições climáticas e da velocidade do vento que não é constante, dificultando o monitoramento e coleta de informações para determinar a eficiência de um protótipo.

Entretanto a montagem de um túnel de vento requer diversos componentes, sendo que alguns podem ser dimensionados como, por exemplo os exaustores, porém outros precisam ser projetados pois não existem no mercado com as especificações necessárias à aplicação, como a parte estrutural que necessita atender rigorosamente as dimensões de projeto para fornecer um resultado de teste confiável. 
Contudo a resposta tecnológica que um aerogeador precisa para ser mais eficiente do ponto de vista aerodinâmico depende de um túnel de vento completo e bem projetado, fornecendo assim uma oportunidade de desenvolvimento de tecnologia compatível com a demanda atual acelerando os resultados e obtendo o máximo desempenho, pois oportuniza o teste de laboratório que promove correções e ajustes essenciais, que seriam muito lentos no ambiente aberto e não controlado.

\section{MATERIAIS E MÉTODOS}

Embora a tecnologia dos métodos computacionais tenha melhorado ao longo do tempo, milhares de horas de testes em túnel de vento ainda são essenciais para o desenvolvimento de uma nova aeronave, turbina eólica ou qualquer outro projeto que envolve interações complexas com o fluxo de ar. Consequentemente, devido ao crescente interesse de outros ramos da indústria e da ciência em aerodinâmica de baixa velocidade, e devido à incapacidade persistente de alcançar soluções precisas com códigos numéricos, túneis de vento de baixa velocidade são essenciais e insubstituíveis durante a pesquisa e design [2].

\subsection{HISTÓRICO DO TÚNEL DE VENTO}

Tudo começou em meados de 1900 quando os irmãos Wilbur Wright e Orville Wright em conjunto com uma série de outros estudiosos iniciaram a construção de protótipos de aeronaves, que inicialmente não eram motorizadas, mas passaram a contar com algum tipo de propulsão, podendo então alçar voo de forma autônoma. No entanto alguns anos antes diversos estudiosos incluindo os irmãos Wright já estavam matematicamente definido os parâmetros e registrando em papeis tudo o que estavam produzindo e projetando [3].

Foi então que em 1901 os irmãos Wright construíram o primeiro túnel de vento para realizar teste em seus recém projetados perfis aerodinâmicos de asas, podendo assim assegurar com melhor eficácia os resultados de seus dimensionamentos [3]. 


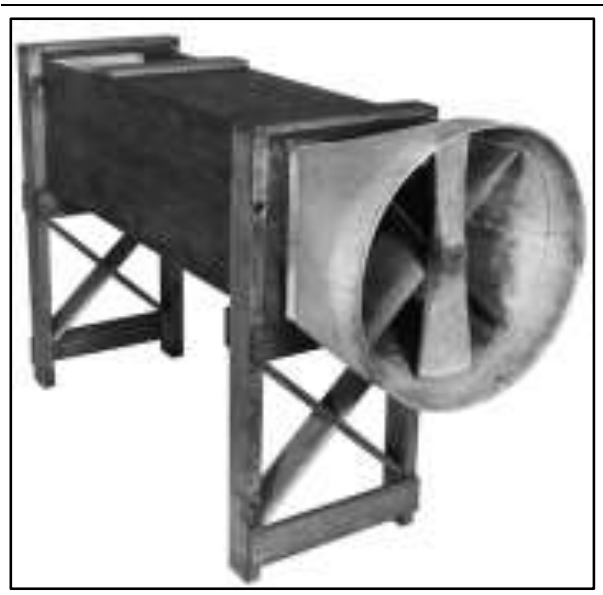

Figura 1. Wright Wind Tunnel [3]

Mais tarde todo o esforço de pesquisa dos irmãos Wright iria se tornar uma agencia do governo dos Estados Unidos denominada NACA, que padronizou todas as normas de aviação e serve de referência ainda hoje para o mundo todo, além disso, a NACA é a base sob a qual foi construída a NASA, uma das mais importantes agencias de pesquisa aeroespacial do mundo [3].

\subsection{CLASSIFICAÇÃO DOS TÚNEIS DE VENTO}

Existem algumas configurações básicas para túneis de vento, estas são classificações quanto à velocidade do ar e forma construtiva do túnel, contudo os túneis de vento podem ter configurações bastante especificas de acordo com a finalidade do teste [4].

\subsubsection{Circuito fechado}

Esta é uma configuração que tem um custo operacional mais baixo, possui um porte geralmente media a grande, pois o ar circula dento do túnel de forma cíclica permanecendo confinado economizando energia, permitindo que sejam aplicados testes com maiores variações de pressão [4].

\subsubsection{Circuito aberto}

A entrada recebe ar da atmosfera e também trabalha na com pressão relativa às dimensões e a velocidade do ar, são túneis de baixa velocidade com a 
finalidade de ensaiar objetos de pequeno a médio porte, necessita também de sistemas de produção de fluxo de ar com boa potência geralmente com variação de velocidade [4].

\subsubsection{Circuitos especiais}

Entretanto existem ainda muitas possibilidades de formato de túneis de vento que são desenvolvidos para atender a propósitos mais específicos, então sendo nestes casos com configurações projetadas mais particularmente ao objeto ensaiado [4].

\section{3 CLASSIFICAÇÃO QUANTO A VELOCIDADE}

Segundo Santos et al. [5], os túneis de vento podem ser caracterizados também quanto à velocidade do fluxo de ar na seção de testes, que é influenciada pelo tipo de circuito do túnel e também pelo tamanho do objeto a ensaiar:

\subsubsection{Túnel de vento de baixa velocidade}

Túnel de vento de baixa velocidade ou subsônico é geralmente aplicado em laboratórios educacionais pelo baixo custo, em termos técnicos a velocidade não pode ultrapassar $135 \mathrm{~m} / \mathrm{s}$ nesta classificação, com um número de Mach até 0,4 [5].

\subsubsection{Túnel de vento transônico}

Pode alcançar uma velocidade do ar de até $340 \mathrm{~m} / \mathrm{s}$ com número de Mach até 1 , sendo utilizados principalmente nas pesquisas aeronáuticas porque a velocidade é compatível com a do voo das aeronaves [5].

\subsubsection{Túnel de vento supersônico}

Apresenta número de Mach de 1 até 5 e velocidade do vento de $340 \mathrm{~m} / \mathrm{s}$ até $1700 \mathrm{~m} / \mathrm{s}$ devido a forma construtiva que utiliza bocais convergentes, o consumo de energia é bastante considerável devidas velocidade que pode ser atingidas [5]. 


\subsubsection{Túnel de vento hipersônico}

Túnel de vento hipersônico possui em sua forma construtiva bocais divergentes e pode atingir velocidades altíssimas entre Mach 5 até 15, ou seja, velocidades acima de $1700 \mathrm{~m} / \mathrm{s}$ [5].

\section{4 COMPONENTES DO TÚNEL DE VENTO}

O túnel de vento é dividido em diversas partes, cada uma delas tem um propósito específico no fluxo do ar e exerce um efeito sobre a qualidade do vento, sempre buscando melhorar as condições de teste [1].

\subsubsection{Seção de teste}

A seção de teste é o componente do túnel de vento onde os objetos são posicionados a fim de testá-los, sua geometria pode variar bastante dependendo da finalidade dos experimentos, podendo ser quadrada, retangular ou circular, contudo, geralmente a forma mais utilizada é retangular por apresentar a melhor forma de acomodação dos objetos [2].

\subsubsection{Difusor}

O difusor tem a finalidade de reduzir a velocidade do ar com o mínio de perdas possíveis, levando em consideração que a perda de velocidade está associada ao ganho de pressão, a redução da velocidade deve ocorrer no menor espaço possível tendo levado em consideração que a variação da pressão e alteração da velocidade pode causar vibrações e perdas consideráveis de carga [6].

\subsubsection{Telas}

O difusor tem a finalidade de reduzir a velocidade do ar com o mínio de perdas possíveis, levando em consideração que a perda de velocidade está associada ao ganho de pressão, a redução da velocidade deve ocorrer no menor espaço possível tendo levado em consideração que a variação da pressão e alteração da velocidade pode causar vibrações e perdas consideráveis de carga [6]. 


\subsubsection{Colmeias}

A colmeia tem a função de corrigir o fluxo na entrada do ar, tornando o mais laminar possível, ou seja, quando o ar passa pela colmeia ela corrige o fluxo que passa através dela para que os filamentos de ar se tornem paralelos, ficando então um escoamento com menos turbulência até que o ar chegue até a câmara de estabilização [4].

\subsubsection{Bocal de contração}

Cone ou bocal de contração faz a ligação entre a câmara de estabilização e a seção de testes aumentando a velocidade média do ar por um fator de escala que pode ultrapassar a 20, contudo, geralmente o fator fica na faixa entre 6 até 10 [4].

\subsubsection{Câmara de estabilização}

A câmara de estabilização tem a finalidade de melhorar a qualidade do fluxo de ar logo após passar pelo difusor, é composta por diversas colmeias que alinhadas produzem a estabilização do fluxo necessário, também podem ser utilizadas telas que ajudam no controle da turbulência do escoamento [6].

\subsubsection{Sistema propulsor}

O sistema propulsor é o responsável por gerar fluxo de ar no túnel de vento, ele deve atender a vazão necessária e vencer as perdas de carga no sistema [6]. Podem ser utilizados um ou mais exaustores conforme sua capacidade, e também a necessidade da vazão do túnel de vento, contudo a turbulência se mostra um pouco diferente quanto ao número de exaustores utilizados, porém, seus efeitos não alteram o fluxo de ar na seção de testes quando empregada uma câmara de estabilização bem dimensionada [1].

\subsection{PERDA DE CARGA}

A perda de carga total no sistema é determinada pelo somatório de perdas de cada componente que compõe o túnel de vento, com exceção do exaustor que fornece energia, sendo assim, as perdas são determinadas pelas variações de 
pressão em função do coeficiente de atrito e da equação do número de Reynolds que leva o diâmetro hidráulico em consideração [4].

\section{RESULTADOS E DISCUSSÃO}

Na etapa inicial um estudo de como funcionam e quais os parâmetros de projeto do circuito do túnel de vento foi realizado, sendo que entre as possíveis configurações foi selecionado o tipo de túnel em circuito aberto, pois economicamente é mais viável por ser muito menor que o de circuito fechado.

Durante a pesquisa de campo foi verificado que a demanda é testar pequenas turbinas tanto montadas quanto apenas uma de suas pás, ficando definido que o tamanho da seção de testes deveria ser de 1,5 × 1,5 × 3 metros, podendo atingir até $15 \mathrm{~m} / \mathrm{s}$ que foi o parâmetro utilizado nos cálculos, caracterizando assim o projeto como túnel do tipo subsônico.

Sendo assim, de posse de todos os requisitos de projeto, o dimensionamento foi efetuado calculando as dimensões de cada componente, no entanto, durante esta etapa muitos componentes tiveram alguma de suas características dimensionais definida pelo projetista, pois são levados em consideração fatores empíricos, tais como espaço disponível e a demanda de fluxo do ar, porém com base na bibliografia pesquisada optou-se pelas melhores configurações de projeto.

Inicialmente foram calculadas as dimensões de cada componente, obtendo na seção de testes as dimensões de 1,5 × 1,5 × 3 m, na entrada do bocal de contração $2 \times 2 \times 1,5$ m e na saída o bocal se conecta a seção de testes, a colmeia com $2 \times 2 \times$ 0,099 m e a cada tela com 0,3 m de comprimento ficando as 4 telas juntas com $2 \times$ $2 \times 1,2$ m, a junção das telas com a colmeias formam a câmara de estabilização com $2 \times 2 \times 1,299 \mathrm{~m}$, o difusor com a entrada do mesmo tamanho que a seção de testes e a saída com 2,024 ×2,024 × $3 \mathrm{~m}$.

Com base nas medidas de cada parte foram determinadas as perdas de carga por componente obtendo na seção de testes $4,804 \mathrm{~N} / \mathrm{m}^{2}$, no bocal de contração $0,824 \mathrm{~N} / \mathrm{m}^{2}$, na câmara de estabilização $0,548 \mathrm{~N} / \mathrm{m}^{2}$, na colmeia 24,292 $\mathrm{N} / \mathrm{m}^{2}$, nas telas $1652,162 \mathrm{~N} / \mathrm{m}^{2}$, no difusor $0,727 \mathrm{~N} / \mathrm{m}^{2}$, resultando em uma perda de carga total de $1683,357 \mathrm{~N} / \mathrm{m}^{2}$. 
Portanto como resultado do dimensionamento foi verificado que instalar apenas um exaustor seria insuficiente devido a sua dimensão, então a carga foi dividia em quatro partes, sendo que cada exaustor deve ter uma potência no eixo igual ou superior a 15,4 kW fornecendo a vazão volumétrica de $8,438 \mathrm{~m}^{3} / \mathrm{s}$ nas condições máximas de operação, que somado as vazões devem produzir deslocamento de ar com velocidade de $15 \mathrm{~m} / \mathrm{s}$ na seção de teste.

Uma vez definidos todos os parâmetros e também finalizado o dimensionamento, foi elaborado um desenho em software 3D SolidWorks ${ }^{\circledR}$, atendendo as especificações das dimensões calculadas de cada componente que foram obtidas através dos cálculos.

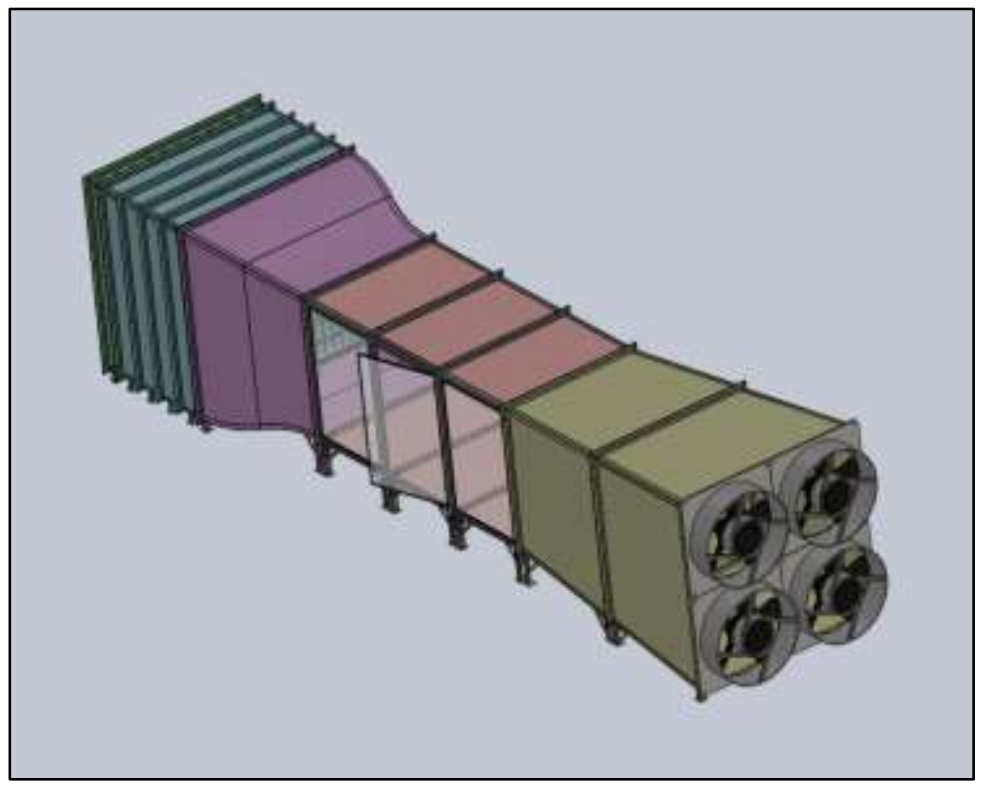

Figura 2. Túnel de vento projetado

Para facilitar uma possível construção, foi adotado como método de projeto a subdivisão de cada componente em uma forma construtiva individual, sendo fixada por meio de parafusos uma parte a outra através dos flanges, cada componente tem sua própria base e pode ser facilmente montado ou desmontado. 


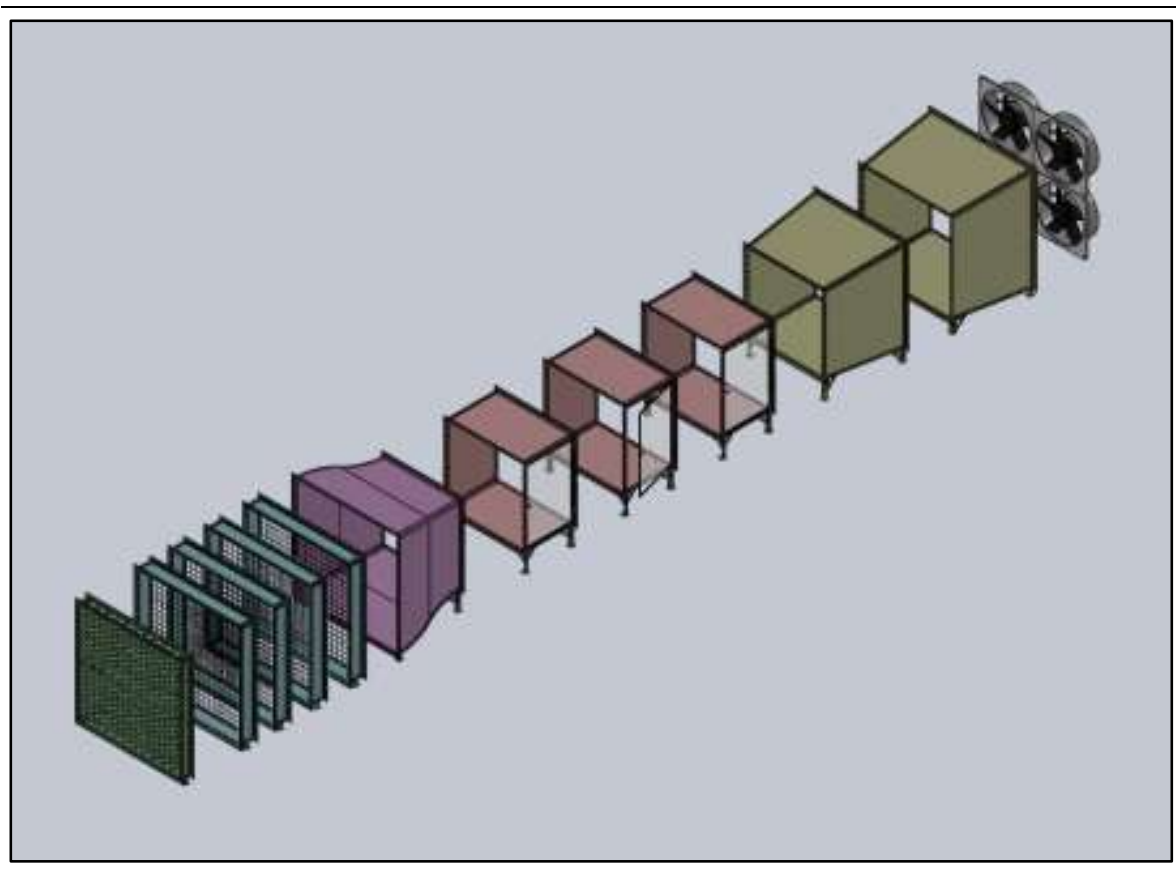

Figura 3. Túnel de vento vista explodida

\section{CONCLUSÕES}

Foram definidos os parâmetros de projeto para testar os modelos de pequeno a médio porte, com a seção de testes de $3 \mathrm{~m}$ de comprimento e difusor com $3 \mathrm{~m}$ e de comprimento velocidade do ar de $15 \mathrm{~m} / \mathrm{s}$, utilizando como parâmetros dos cálculos do ar a pressão e temperaturas padrão.

A seção de testes teve as suas dimensões definidas de acordo com a pesquisa de campo, cujo resultado foi a definição da seção de testes de 1,5 ×1,5 m, cujos valores atendem a demanda necessária.

O túnel de vento tem um princípio de funcionamento relativamente simples, contudo são necessários diversos cálculos para determinar as perdas de carga, pois todos os fatores influenciam na velocidade de escoamento e pressão dinâmica. O dimensionamento foi realizado calculando as áreas e os diâmetros hidráulicos de cada componente separadamente, bem como o comprimento e cálculos específicos de cada parte, a pressão e a perda de carga também foram determinadas com base nas dimensões e nas propriedades físicas do ar. Uma vez 
que todas as partes foram dimensionadas, a perda de carga total foi determinada e a potência necessária para colocar o túnel em funcionamento foi determinada, devendo esta ser de no mínimo 15,4 kW no regime máximo de trabalho.

\section{REFERÊNCIAS}

[1] OKAMOTO, Satoru; MERRISON, Jonathan; PORTMAN, V.; SANDLER, B.; CHAPSKY, V.; LIU, Xin; QIU, Yuanying; DUAN, Xuechao; BENEDETTO, Sara Di; RUFOLO, Giuseppe C.; MARINI, Marco; TRIFONI, Eduardo; ANDRIANNE, Thomas; RAZAK, Norizham Abdul; DIMITRIADIS, Grigorios; KOTHERA, Curt S.; WERELEY, Norman M. Wind Tunnels. Croatia: InTech, c2011.

[2] HERNÁNDEZ, Miguel A. González; LÓPEZ, Ana I. Moreno; JARZABEK, Artur A.; PERALES, José M. Perales; WU, Yuliang; XIAOXIAO, Sun. Design Methodology for a Quick and Low-Cost Wind Tunnel. Polytechnic University of Madrid, Spain \& Beijing Institute of Technology, China, 2013. Disponível em:

$<$ http://www.intechopen.com/books/wind-tunnel-designs-and-their-diverseengineering-applications/design-methodology-for-a-quick-and-low-cost-windtunnel> Acessado em: 08/09/2016.

[3] NASA. Learning to Fly: The Wright Brothers' Adventure.2002. Disponível em: $<$ https://www.nasa.gov/pdf/58225main_Wright.Brothers_508.pdf> Acessado em 12/09/2016.

[4] BARLOW, Jewel B.; RAE, William H. Jr.; POPE, Alan. Low-Speed Wind Tunnel Testing. 3. ed. New York: John Wiley \& Sons, 1999.

[5] SANTOS, Adalberto Rodrigo dos; SILVA, Bruno Washington Xavier da; NETO, Francisco Santiago; LOPES, Laura Dias; DIONÍSIO, Thais Honório. Elaboração de túnel de vento para aplicações de ensaios aerodinâmicos. Instituto Federal De Educação, Ciência e Tecnologia de São Paulo. Campus São José Dos Campos, 2014. Disponível em: $<$ http://sjc.ifsp.edu.br/biblioteca/index.php/component/phocadownload/ category/6-mecanica?download=4:elaboracao-de-tunel-de-vento-para-aplicacoesde-ensaios-aerodinamicos > Acessado em: 13/09/2016.

[6] COUTINHO, Felipe Rodrigues. Projeto de um Túnel de Vento Subsônico do Tipo Soprador. Universidade Federal do Rio de Janeiro, 2014. Disponível em: <http://monografias.poli.ufr.br/monografias/monopoli100 12170.pdf> Acessado em: 08/09/2016. 